\title{
Interaction on an Additive Scale
}

\author{
Renée de Mutsert ${ }^{\mathrm{a}}$ Dinanda J. de Jager ${ }^{\mathrm{a}}$ Kitty J. Jager ${ }^{\mathrm{b}}$ Carmine Zoccali ${ }^{\mathrm{c}}$ \\ Friedo W. Dekker a, b \\ a Department of Clinical Epidemiology, Leiden University Medical Centre, Leiden, and ${ }^{\mathrm{b}}$ ERA-EDTA Registry, \\ Department of Medical Informatics, Academic Medical Center, University of Amsterdam, Amsterdam, \\ The Netherlands; 'CNR-IBIM Clinical Epidemiology and Pathophysiology of Renal Diseases and Hypertension, \\ Renal and Transplantation Unit, Ospedali Riuniti, Reggio Calabria, Italy
}

\section{Key Words}

Epidemiology $\cdot$ Risk factors $\cdot$ Joint exposure $\cdot$ Interaction

\begin{abstract}
Many clinical epidemiological studies investigate whether a certain exposure, or risk factor, is associated with the incidence of disease or mortality. It may be of interest to study whether this association is different in different types of patients, or to study joint effects. To investigate whether the effect of one risk factor differs across the strata of another risk factor, the presence of interaction among two risk factors can be examined. In statistics, interaction refers to the inclusion of a product term of two risk factors in a statistical model. Statistical interaction thereby evaluates whether the association deviates from either additivity or multiplicativity, depending on the scale of the model. From a public health perspective, the assessment of interaction on an additive scale may be most relevant. For a transparent presentation of interaction effects, it is recommended to report the separate effect of each exposure as well as the joint effect compared to the unexposed group as a joint reference category to permit evaluation of interaction on both an additive and multiplicative scale.

Copyright $\odot 2011$ S. Karger AG, Basel
\end{abstract}

\section{Interaction between Risk Factors}

Many clinical epidemiological studies investigate whether exposure, or a risk factor, is associated with the development or progression of disease or mortality. It may be of interest to study whether this association is different in different types of patients. For example, we might want to know whether the observed association between exposure to life style risk factors and the incidence of chronic kidney disease (CKD) differs between men and women [1]. Besides observational studies, randomized clinical trials commonly evaluate whether treatment effects differ across certain subgroups. For example, the Cathedia study randomized 750 critically ill patients requiring acute renal replacement therapy to receive jugular or femoral vein catheterization, and studied if rates of infectious complications differed in predefined subgroups of catheter insertion time, BMI, sex, and type of renal replacement therapy [2]. In order to address such research questions, it can be examined whether the effect of one risk factor on a certain outcome differs across strata of another risk factor. In other words, the presence of interaction can be examined.

In the literature, interaction is studied and reported in several different ways $[3,4]$. A literature survey concluded 


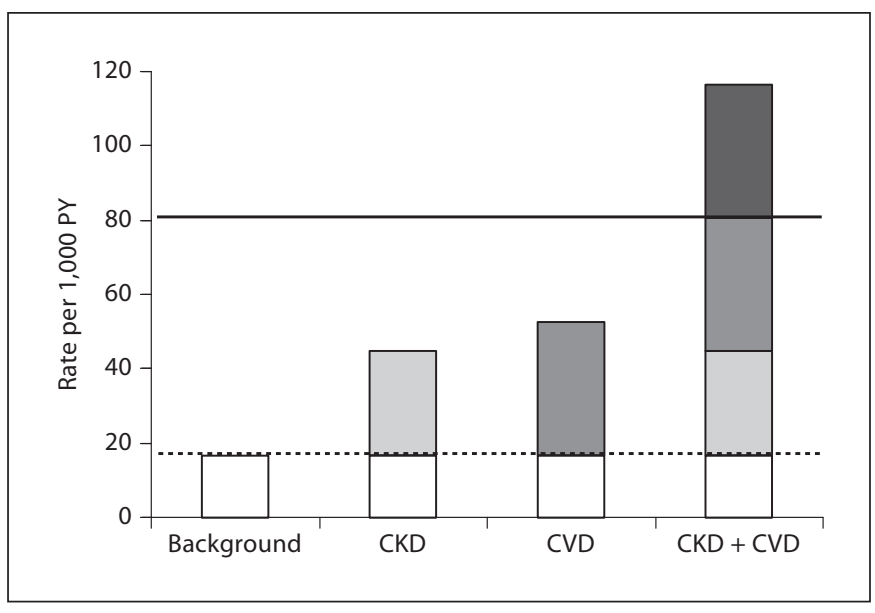

Fig. 1. Incidence rates of composite outcome per 1,000 PY in 26,147 individuals during 10 years of follow-up without CKD or CVD, with CKD or CVD or with both CKD and CVD at baseline [9]. The dotted line indicates the background rate (16.9/1,000 PY); the straight line indicates exact additivity of effects.

that the reporting of interaction in the medical and epidemiological literature is inadequate [3]. One common mistake is the comparison of $\mathrm{p}$ values across subgroups and to conclude that interaction is present when there is a statistically significant association in one category (for example, men) but not in the other (women) $[3,5]$. This paper illustrates to what extent different approaches of the concept interaction can result in different answers to a research question and shows how interaction can be examined on an additive scale using incidence rates.

\section{Statistical Interaction}

In statistics, interaction refers to the inclusion of a product term of two risk factors in a statistical model for a better fit of the model [6]. Statistical interaction thereby evaluates whether the association deviates from either additivity or multiplicativity, depending on the scale of the model. In this way, the presence of interaction and its interpretation depend on the underlying scale of the model. For example, in a linear regression model, statistical interaction indicates that the association between the two risk factors and the outcome under study is no longer additive (for example, the interaction between salt intake and age in the association with blood pressure as a continuous outcome variable). In contrast, logistic and Cox regression models are multiplicative models. Statistical interaction in such models (for example, the interaction between salt intake and age in association with hypertension as a dichotomous outcome variable) indicates a deviation from multiplicativity, rather than from additivity. This may not be a problem as long as the researcher is aware that the choice of the statistical model and underlying scale has an impact on the interpretation of the interaction effect. However, from a public health perspective, the assessment of interaction on an additive scale may be most relevant [5-7].

\section{Interaction on an Additive Scale}

A departure from risk additivity implies that the number of cases attributable to the combined effect of two risk factors is more or less than the sum of the number of cases that would be caused by each risk factor separately. In the absence of bias, departures from risk additivity imply that some subgroups would obtain a greater absolute risk reduction from the intervention than others would [6-8]. Therefore, the additive scale, which uses absolute risks, may be most relevant for public health and clinical decision making [5].

\section{Example 1}

Because CKD is a risk factor for cardiovascular disease (CVD) but CVD may also promote CKD, it can be hypothesized that CKD and CVD have a synergistic effect on future cardiovascular and mortality outcomes. With data from a cohort study in 26,147 individuals from 4 community-based studies [9] the presence of additive interaction between CKD and CVD in the association with a composite outcome of cardiac events, stroke and death can be examined. Figure 1 shows the incidence rates of the composite outcome in 10 years of follow-up [9]. In persons without CKD and CVD at baseline, the rate of the composite outcome was 16.9/1,000 person-years (PY). This can be considered as the background rate: from all persons without CVD and CKD at baseline, 16.9/1,000 PY had a cardiac event or died within 10 years. In persons with CKD at baseline, this was 44.9/1,000 PY, resulting in a risk difference of $44.9-16.9=28 / 1,000 \mathrm{PY}$. In the absence of bias, this additional 28/1,000 PY can be attributed to exposure to CKD. In persons with CVD at baseline, the incidence rate was 52.7/1,000 PY, $52.7-16.9=$ $35.8 / 1,000 \mathrm{PY}$ more as a result of exposure to CVD. If no 
interaction between CKD and CVD was present, we would expect an outcome rate in persons exposed to both CKD and CVD at baseline of $16.9+28+35.8=80.7 / 1,000$ PY: 16.9/1,000 PY will have the outcome anyway, an extra $28 / 1,000 \mathrm{PY}$ as a result of exposure to CKD and an additional 35.8/w1,000 PY as a result of exposure to CVD. The observed rate, however, was 116.4/1,000 PY [9]. Thus, the composite outcome occurred in $116.4-80.7=35.7$ persons/1,000 PY more than we would expect on the basis of the sum of the separate effects of CVD and CKD (fig. 1).

This more than additive effect means that, as a result of interaction between CKD and CVD, an excess risk of 35.7 cases/1,000 PY has been observed. Assuming causality, this implies that if the occurrence of CKD could be prevented in the general population, this would not only prevent the 28 cases/1,000 PY, but in addition the 35.7 cases/1,000 PY would be prevented that would have occurred because of an interaction between CKD and CVD. In contrast, when examined on a multiplicative scale the combination of CKD and CVD appeared protective in this study $[9,10]$, whereas more patients reached the outcome than was expected on the basis of the separated risks of CKD and CVD. This example shows that by using absolute risks, additive interaction may be considered most relevant because of its potential implications for public health.

\section{Example 2}

In the Cathedia study of 750 critically ill patients the presence of interaction between catheter site and subgroups of BMI has been examined by including a product term of catheter site (femoral or jugular) and BMI (in tertiles) in a Cox proportional hazards regression model [2]. A significant interaction $(\mathrm{p}<0.001)$ was found for the effect of BMI on the association between catheter site and catheter colonization. Inherent to the Cox regression model, this means a deviation from multiplicativity of effects. When the regression coefficient of the product term is not given, the magnitude and the direction of the interaction effect cannot be interpreted from the p value only, nor whether there is interaction on an additive scale. However, the authors also reported absolute incidence rates of catheter colonization in strata of BMI and catheter site (table 1, showing the upper and lower tertiles of $\mathrm{BMI})$. These incidence rates provide the reader with sufficient information to evaluate the presence of interaction on both an additive and multiplicative scale. Patients with a low BMI $(<24.2)$ had a higher incidence of colo-
Table 1. Incidence rates of catheter colonization per 1,000 catheter-days by femoral or jugular vein catheterization and BMI in 750 critically ill patients requiring acute renal replacement therapy

\begin{tabular}{lll}
\hline Catheter site & BMI & Incidence rate \\
\hline Femoral vein & $<24.2$ & 23.7 \\
Jugular vein & $<24.2$ & 45.4 \\
Femoral vein & $>28.4$ & 50.9 \\
Jugular vein & $>28.4$ & 24.5 \\
\hline
\end{tabular}

nization in the jugular versus femoral group (45.4 vs. $23.7 / 1,000$ catheter-days, respectively). In the femoral group, patients with a high BMI (>28.4) also had a higher incidence of colonization compared with patients with a low BMI (50.9 vs. 23.7 per 1,000 catheter-days, respectively). Finally, patients with a high BMI in the jugular group did not have the expected rate of 72.6/1,000 catheter-days (the background rate 23.7 + risk difference jugular 21.7 + risk difference BMI 27.2 = 72.6/1,000 catheterdays) but 24.5/1,000 catheter-days, implying a less than additive effect. A less than additive effect is also referred to as interaction on an additive scale.

In this example, the interaction effect was both less than additive and less than multiplicative. However, the interpretation of results can differ depending on the scale on which the presence of interaction is examined. The first example showed that it is possible to find a less than multiplicative effect, but a more than additive effect within the same data $[9,10]$. Or, as the next example illustrates, it is also possible to find different effects in subgroups on a multiplicative scale, whereas the effects are similar on an additive scale.

\section{Example 3}

A cohort study of incident dialysis patients (Netherlands Cooperative Study on the Adequacy of Dialysis, NECOSAD) studied the association between self-rated health and mortality and examined the presence of interaction of self-rated health with age [11]. The crude mortality rates and adjusted hazard ratios are shown in table 2 . If we consider the crude effects per age stratum, in patients younger than 65 years of age, mortality rate associated with a poor self-rated health would be almost 20 times higher (24.5/1.4 per 100 PY) than in patients with 
Table 2. Crude mortality rates per $100 \mathrm{PY}$ and adjusted hazard ratios in 1,443 dialysis patients in 7 years of follow-up

\begin{tabular}{llcl}
\hline Age & SRH & $\begin{array}{l}\text { Mortality } \\
\text { rate/100 PY }\end{array}$ & $\begin{array}{l}\text { Hazard ratio } \\
(95 \% \mathrm{CI})\end{array}$ \\
\hline$\geq 65$ years & poor & 35.3 & $10.6(2.5,45.7)$ \\
$\geq 65$ years & good & 9.0 & $4.5(0.9,21.8)$ \\
$<65$ years & poor & 24.5 & $8.0(1.9,34.5)$ \\
$<65$ years & good & 1.4 & 1 (reference) \\
\hline
\end{tabular}

SRH $=$ Self-rated health; $95 \% \mathrm{CI}=95 \%$ confidence interval.

a good self-rated health. In patients of 65 years or older, the mortality rate associated with poor self-rated health would be almost 4 times higher (35.3/9 per $100 \mathrm{PY}$ ) than in patients with a good self-rated health. On the basis of these stratum-specific relative risks, self-rated health seems more strongly associated with mortality in younger than in older dialysis patients. However, the absolute mortality rate among older patients with an excellent selfrated health is much higher than among younger patients (9.0 and 1.4/100 PY, respectively). On an additive scale, the effects of having a poor self-rated health appear similar for the younger (23.2 extra cases/100 PY) and older patients (26.3 extra cases/100 PY).

\section{Conclusion}

The interpretation of statistical interaction is not consistent. From a public health perspective, the assessment of interaction on an additive scale may be most relevant. When using product terms in statistical models, one should consider whether the underlying scale of the mod$\mathrm{el}$ is additive or multiplicative. Since logistic regression is the most commonly used model in clinical research, the majority of analyses of epidemiologic data are performed on a multiplicative scale. An accompanying paper in this series presents surrogate measures of additive interaction that can be used to evaluate interaction on an additive scale, using effect measures derived from multiplicative models [12]. For a transparent presentation of interaction effects it is recommended to report the separate effect of each exposure as well as the joint effect, each relative to the unexposed group as joint reference category (as shown in tables 1,2$)[5,13]$. This will provide the reader with sufficient information to evaluate the presence of interaction on both an additive and multiplicative scale [5].

\section{Disclosure Statement}

There are no conflicts of interest.

\section{References}

1 Hallan S, de Mutsert R, Carlsen S, et al: Obesity, smoking, and physical inactivity as risk factors for CKD: are men more vulnerable? Am J Kidney Dis 2006;47:396-405.

-2 Parienti JJ, Thirion M, Mégarbane B, et al: Femoral vs jugular venous catheterization and risk of nosocomial events in adults requiring acute renal replacement therapy. JAMA 2008;299:2413-2422.

-3 Knol MJ, Egger M, Scott P, Geerlings MI, Vandenbroucke JP: When one depends on the other: reporting of interaction in casecontrol and cohort studies. Epidemiology 2009;20:161-166.

4 Ahlbom A, Alfredsson L: Interaction: a word with two meanings creates confusion. Eur J Epidemiol 2005;20:563-564
5 Vandenbroucke JP, von Elm E, Altman DG, et al: Strengthening the Reporting of Observational Studies in Epidemiology (STROBE): explanation and elaboration. PLoS Med 2007;4:e297.

6 Greenland S, Lash TL, Rothman KJ: Concepts of interaction; in Modern Epidemiology. Philadelphia, Lippincott Williams \& Willkins, 2008, pp 71-83.

7 Rothman KJ: Measuring interactions; in Epidemiology: An Introduction. New York, Oxford University Press, 2002, pp 168-180.

-8 Greenland S: Interactions in epidemiology: relevance, identification, and estimation. Epidemiology 2009;20:14-17.

-9 Weiner DE, Tabatabai S, Tighiouart H, et al: Cardiovascular outcomes and all-cause mortality: exploring the interaction between CKD and cardiovascular disease. Am J Kidney Dis 2006;48:392-401.
10 de Mutsert R, Jager KJ, Zoccali C, Dekker FW: The effect of joint exposures: examining the presence of interaction. Kidney Int 2009; 75:677-681

11 Thong MS, Kaptein AA, Benyamini Y, Krediet RT, Boeschoten EW, Dekker FW, Netherlands Cooperative Study on the Adequacy of Dialysis (NECOSAD) Study Group: Association between a self-rated health question and mortality in young and old dialysis patients: a cohort study. Am J Kidney Dis 2008;52:111-117.

12 de Jager DJ, de Mutsert R, Jager KJ, Zoccali C, Dekker FW: Reporting of interaction. Nephron Clin Pract, submitted.

13 Botto LD, Khoury MJ: Commentary: facing the challenge of gene-environment interaction: the two-by-four table and beyond. Am J Epidemiol 2001;153:1016-1020. 